Aim of the study: Uterine fibroids are one of the most prevalent benign tumors. This study aimed to evaluate the effect of uterine artery embolization on treating and reducing the size of symptomatic uterine fibroids.

Material and methods: Eighty patients with uterine fibroids were selected for this study. Then ultrasound and Doppler were performed by a radiologist to evaluate the size, number, echogenicity, vascularity, and location of fibroids in the uterine wall. Before performing uterine artery embolization, prothrombin time, partial thromboplastin time, international normalized ratio, blood urea nitrogen, and creatinine tests were performed for all the patients to identify background problems. Finally, SPSS 22 was used for data analysis.

Results: The dominant fibroid volume before the embolization was $244.57 \mathrm{~cm}^{3}$, which decreased to $219.96 \mathrm{~cm}^{3}, 190.58 \mathrm{~cm}^{3}, 114.18 \mathrm{~cm}^{3}$ $140.51 \mathrm{~cm}^{3}$, and $78.86 \mathrm{~cm}^{3}$ in the first week, first month, third month, sixth month and first year after embolization, which was statistically significant $(p<0.001$ in all cases). Uterine volume in multiple tumor before the embolization was $486.27 \mathrm{~cm}^{3}$ which decreased to $408.36 \mathrm{~cm}^{3}$, $387.60 \mathrm{~cm}^{3}, 299.67 \mathrm{~cm}^{3}, 190.00 \mathrm{~cm}^{3}$ and $172.33 \mathrm{~cm}^{3}$ after the first week, first month, third month, sixth month and first year after embolization, which in the first week and third month was statistically significant and not significant in other cases $(p=0.003, p=0.500, p=0.028$, $p=0.068, p=0.109)$. The relationships of the number of fibroid tumors, echogenicity and vascularity with volume reduction were not statistically significant $(p=0.924, p=0.208$, $p=0.455$ ).

Conclusions: Uterine artery embolization is an effective treatment for fibroid tumors. In this study, the number of tumors, echogenicity, and vascularity of tumors had no effect on tumor volume reduction.

Key words: grayscale ultrasound, Doppler, uterine fibroids, uterine artery embolization.

Contemp Oncol (Pozn) 2019; 23 (1): 32-36 DOI: https://doi.org/10.5114/wo.2019.84111

\section{Impact of grayscale and Doppler ultrasound characteristics on reducing the size of tumors in the treatment of uterine fibroids by uterine artery embolization}

\author{
Azim Motamedfar ${ }^{1}$, Mohammad Momen Gharibvand ${ }^{1}$, Said Tamiz ${ }^{2}$
}

${ }^{1}$ Department of Radiology, Golestan Hospital, Ahvaz Jundishapur University of Medical Sciences, Ahvaz, Iran

${ }^{2}$ Student Research Committee, Ahvaz Jundishapur University of Medical Sciences, Ahvaz, Iran

\section{Introduction}

Today one of the biggest health issues is cancer and tumor management and treatments [1-3]. Uterine fibroids are one of the most prevalent benign tumors, and are observed in about $40 \%$ of women up to 35 years old [4]. Fibroids are considered as one of the major problems of the health system; $30 \%$ of the 600,000 hysterectomies in the United States are caused by these tumors [5]. Most women with fibroids have no symptoms; however, some of them have troubling symptoms [6]. These symptoms are divided into 4 categories: bleeding symptoms (irregular or severe menstruation bleeding), pain (in back and pelvis), sign of tumor (pressure on bladder and intestine and its increased movements), and decreased fertility [7]. In the United States, women with fibroids have 3.2 times higher health costs than healthy women [6]. Also in this country, 2 billion dollars is annually spent on treatment of fibroids [8].

Diagnosis of fibroid tumors is accompanied by clinical uncertainty in examination and observing a big uterus, and it is confirmed by ultrasound of the pelvis. In diagnosing fibroids, ultrasound is as effective as magnetic resonance imaging (MRI), although $\mathrm{MRI}$ indicates the tumors with higher resolution. The common treatments for fibroids include hysterectomy, myomectomy, and hormone therapy, which have various advantages and disadvantages. Less invasive therapy includes uterine artery embolization [5]. Uterine artery embolization has been recognized as a treatment for uterine fibroids since 1995 [9]. In this method, the purpose is to transfer specific particles (polyvinyl alcohol or gelatin-coated polymer microspheres) to both arteries of the uterus, creating obstruction and ischemia in the fibroid without damaging other parts of the uterus [10-12]. In many studies, after performing uterine artery embolization, menorrhagia has been improved in 85-95\% of patients and pressure signs of the tumor have been improved in 70-90\% of patients [13]. Also in another study, an average size reduction of 57/8\% (amount of reduction in comparison to the basic size) in fibroid and an average size reduction of $39 / 4 \%$ in the uterus over 12 months were observed [14].

Different factors influence the uterine artery embolization. In a study, it was found that the base diameter of fibroid is not a risk factor for performing uterine artery embolization [13]. However, other studies have suggested that big fibroids are a relative contradictor of performing uterine artery embolization and they increase the risk of infection, sepsis, uterine necrosis, and death [15]. Also, it has been suggested that uterine artery embolization should not be performed in big fibroid tumors ( $\geq 10 \mathrm{~cm}$ in diameter) [16]. 
Since there is not any specific guideline for uterine artery embolization, preparation of an indication and treatment strategy for uterine artery embolization is necessary for adoption of an appropriate and integrated approach by gynecologists and radiologists [17]. This study aimed to evaluate the effect of uterine artery embolization on treating and reducing the size of symptomatic uterine fibroids in Ahvaz, and to investigate whether the number, size, echogenicity, and vascularity of fibroids influence the treatment by uterine artery embolization.

\section{Material and methods}

After obtaining permission from ethics committee of the Ahvaz Jundishapur University of Medical Sciences, Ahvaz, Iran, 80 patients referring to the radiology wards of the two hospitals of Imam Khomeini and Golestan in Ahvaz in the years 2014 and 2015 were selected and entered into the study. The patients had been referred to the hospital by a gynecologist for treatment of symptomatic fibroids, and gynecological examination was performed for all of them. The criterion for participation in the study was a symptomatic fibroid in the patients who had refused to take medicine, or medical treatment for them had failed and they refrained from hysterectomy. Pregnant women or people with endometrial hyperplasia, different types of carcinoma (cervix, uterus, and ovary), pelvic inflammation, pedunculated myomas, endometriosis, and adenomyosis did not participate in this study.

The patients were asked for demographic information. Also, all of them received the necessary information about the advantages and side effects of the treatment method and the alternative treatments. Then ultrasound and Doppler (GE 200; GE, USA; Convex probe, 2.5-5 MHz) were performed by a radiologist to evaluate the size, number, echogenicity, vascularity, and the location of fibroids in the uterine wall. The size of the biggest fibroid tumor and the uterine volume were calculated by the ellipse formula (length $\times$ depth $\times$ 0.5233) .

Before performing uterine artery embolization, prothrombin time (PT), partial thromboplastin time (PTT), international normalized ratio (INR), blood urea nitrogen $(B \cup N)$, and creatinine (CR) tests were performed for all the patients to identify background problems.

During the process, patients receive anesthesia with midazolam and fentanyl citrate. The operating angiography device is a GE DEX DSA with 1200 MA and 140 KVP. For performing embolization, we enter the right femoral artery. The used catheter is a 4 or 5 Fr Cobra catheter and the used embolization material is 300-500 $\mu$ of polyvinyl alcohol (PVA) particles (Contour; Boston Scientific, Boston, MA, USA) which is injected by the radiologist under fluoroscopic guidance.

Before performing uterine artery embolization, patients receive a dosage of venous cefalotin ( $1 \mathrm{~g}$ ) and gentamycin $(80 \mathrm{mg})$. After the embolization process, patients are hospitalized under medical care for 1 day. The patients' pain is controlled by pethidine $(25-50 \mathrm{mg})$ or morphine sulfate (10 mg).
At the time of being released, patients receive ibuprofen (400 mg orally four times daily) and diclofenac sodium (50 mg suppository every $6 \mathrm{~h}$, if necessary) to control their pain. Also, the patients are asked to refer for pursuing the treatment after one week, one month, three months, six months, and one year. At these visits, abdominal ultrasound is performed for the patients to assess the changes of uterus and fibroid size.

After data collection, the Wilcoxon method and repeated data analysis (repeated measurement ANOVA) were used to study the relationship between the variables.

SPSS 22 software was used for obtaining descriptive indicators and drawing the charts.

\section{Results}

Sixty-nine patients (86.3\%) had prevailing fibroid tumors and 11 people (13.8\%) had multiple tumors. Most of the women participating in the study had single fibroids (86.3\%). In the study of the tumors with prevailing fibroids, it was found that 35 patients had 1 tumor (50.7\%), 19 patients had 2-5 tumors (27.5\%) and 15 patients had more than 5 tumors (21.7\%). In the single tumor group, in terms of the number of fibroids, most of the participating women had one fibroid tumor (50.7\%). In the study of the tumors with prevailing fibroid tumors, echogenicity studies showed that 37 patients had hypoechoic tumors (53.6\%), 21 patients had heterogenic tumors (30.4\%), 7 patients had layer tumors (10.1\%), 3 patients had isoechoic tumors (3\%) and 1 patient had a hyperechoic tumor (1.4\%). In the prevailing tumor group, in terms of echogenicity of the tumor, most of the women participating in the study were hypoechoic (53.6\%). In terms of location of the prevailing tumor, 29 patients had tumors in intramural and submucosal areas (42\%), 19 patients had tumors in the transmural area (27.5\%), 8 patients had tumors in the submucosal area (11.6\%), 6 patients had tumors in intramural and subserosal areas (8.7\%), 4 patients had tumors in subserosal (5.8\%), and 3 patients had tumors in intramural areas (4.3\%). In the prevailing tumor group, in terms of location of fibroids, most of the participating women were of the intramural and submucosal type (42\%). In terms of vascular examination of the prevailing fibroid, 34 people were high vascular (49.6\%), 32 people were low vascular (46.6\%) and 3 people had no vascular signal (4.3\%). In the prevailing tumor group, in terms of vascularity, most of the participating women were high vascular (49.3\%).

\section{Study of volume changes \\ Study of volume changes of prevailing fibroid tumor}

In the prevailing tumor group, the average primary volume of the fibroid was $244 / 57 \mathrm{~cm}^{3}$ (standard deviation $[S D]=378 / 30)$, and in the first week of referring, it was $219 / 96 \mathrm{~cm}^{3}$ (SD = 369/60). In the fourth week, considering the $7(10.1 \%)$ patients who have not referred, the average volume of fibroids was $190 / 58 \mathrm{~cm}^{3}(S D=272 / 74)$ in the prevailing tumor group. In the third month, considering the 21 (30.4\%) patients who have not referred, the average volume of fibroids was $114 / 18 \mathrm{~cm}^{3}(S D=197 / 59)$. In 


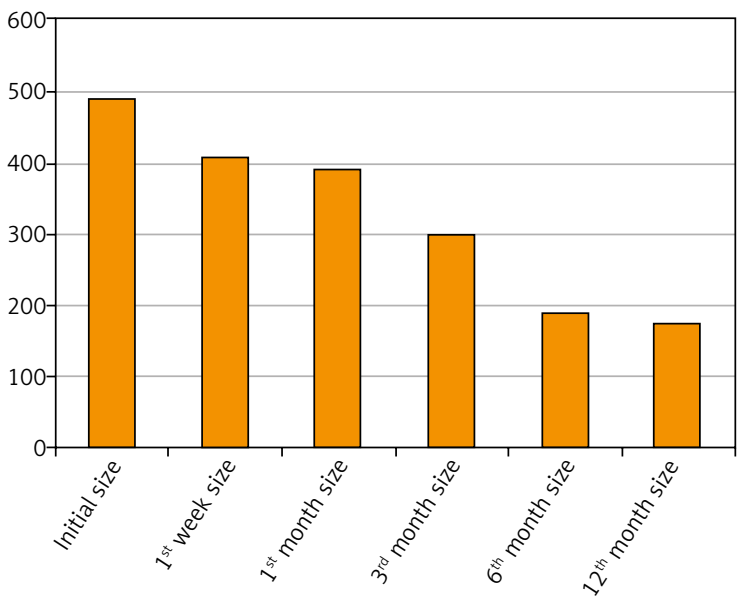

Fig. 1. Trend of changes in mean uterine volume in multiple tumors, which is a non-significant reduction

the sixth month, considering the 34 patients (42/2\%) who have not referred, the average volume of the fibroid was $140 / 51 \mathrm{~cm}^{3}$ (SD = 237/40). In the first year, considering the 47 (68.1\%) patients who have not referred, the average volume of fibroid was $78 / 86 \mathrm{~cm}^{3}(S D=152 / 03)$.

\section{Study of changes of uterus volume in multiple fibroid tumors}

In the multiple tumor group, the average primary volume of the uterus was $486 / 27 \mathrm{~cm}^{3}(\mathrm{SD}=209 / 09)$, and in the first week of referring, it was $408 / 36 \mathrm{~cm}^{3}(\mathrm{SD}=180 / 47)$.

In the fourth week, considering the 6 patients (54\%) who have not referred, the average uterus volume was $387 / 60 \mathrm{~cm}^{3}$ (SD = 236/61). In the third month, considering the 5 patients (45\%) who have not referred, the average uterus volume in the third month was 299/67 $\mathrm{cm}^{3}$ $(S D=221 / 57)$. In the sixth month, considering the 7 patients (63\%) who have not referred, the average uterus volume was 190/00 $\mathrm{cm}^{3}(\mathrm{SD}=41 / 87)$. In the first year, considering the 8 patients (72\%) who have not referred, the average uterus volume was $172 / 33 \mathrm{~cm}^{3}(\mathrm{SD}=40 / 20)$ (Fig. 1).

\section{Results of Wilcoxon nonparametric tests for studying the two-variable relationship}

The results of Wilcoxon nonparametric test shows that in the prevailing tumor group, the average scores of fibroid volume in the first week, the fourth week, the sixth month, and after one year are significantly different from the primary volume and the previous stage $(p<0.001)$. In other words, the average score of fibroid volume at every time was significantly different from the previous follow-up.

The results of the Wilcoxon nonparametric test show that in the multiple tumor group, the average score of uterus volume in the first week of referring was significantly difference from the primary volume $(p=0.003)$. In other words, the average score of uterus volume in the first week of referring had a statistically significant reduction compared with the score before performing uterine artery embolization. Also, in the third month of referring, it had a significant difference from the primary volume

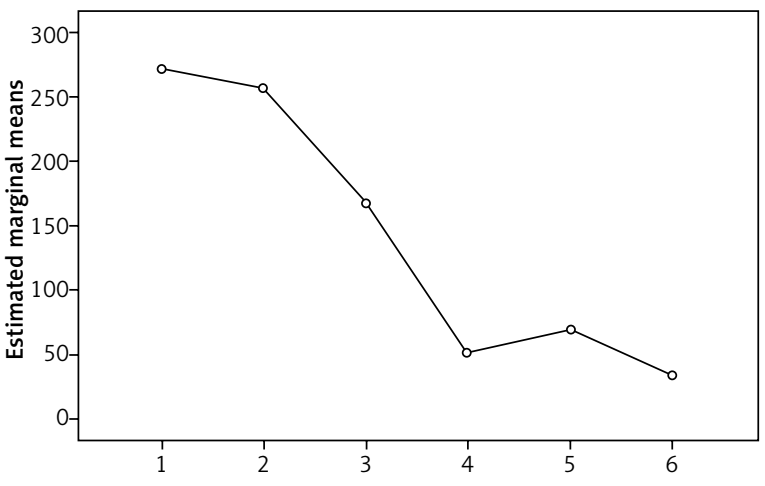

Fig. 2. Trend of changes in mean volume of fibroids at successive measurements

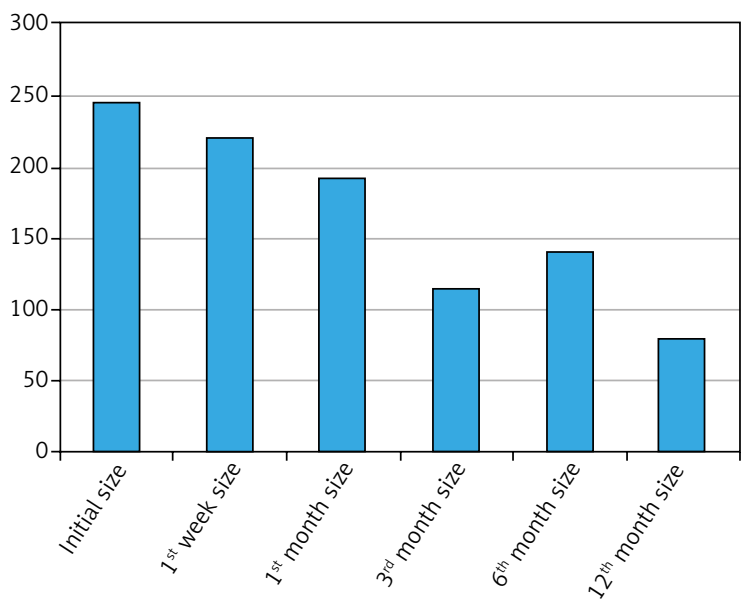

Fig. 3. Process of changes in mean volume of fibroids at successive measurements

$(p=0.28)$. However, no statistically significant difference was observed at other times (Fig. 2).

\section{Results of repeated measurement ANOVA test}

In order to compare the average scores of fibroid volume at different times and controlling the confounding effect of other variables such as place and number of tumors, the repeated measurement ANOVA test was used. The average scores of fibroid volume over repeated measurements (six time points) were significantly different and are significantly descending. In order to study the relationship of the variables of tumor numbers, echogenicity, location, and vascularity with the decrease of the tumor volume, repeated measurement ANOVA was used. Only a significant relationship between the prevailing location of the fibroid tumor and decrease of the tumor volume was observed (Fig. 3).

\section{Discussion}

In our study during the years 2014 and 2015, 80 eligible patients participated, studied, and intervened. Among them, 69 patients (86.3\%) had prevailing fibroid tumors and 11 patients (13.8\%) had multiple tumors. In other studies conducted to investigate the effect of uterine artery embolization on the treatment process, Goodwin et al. studied 60 people, Siskin et al. studied 49 people, Pelage 
et al. studied 80 people, McLucas et al. studied 167 people, Anderson et al. studied 62 people, and Katsumori et al. studied 60 people [18]. Therefore, it seems that the number of patients studied in our research is in an acceptable range. In the prevailing fibroid tumor group, most of the patients $(50.7 \%)$ had one fibroid tumor. In terms of location of the prevailing fibroid in the uterine wall, most of the patients (42.0\%) had tumors in intramural and submucosal and transmural (27.5\%) areas. The results are similar to the results of the research conducted by Firouznia et al. in which most of the studied patients in the prevailing fibroid group (40.5\%) had one tumor and in terms of tumor location, most of the patients $(29.7 \%)$ had tumors in intramural and submucosal areas [13].

In the echogenicity study of prevailing fibroids, most of the tumors (53.6\%) were hypoechoic and heterogeneous (30.4\%). In the study conducted by Weintraub et al. on 14 patients, $14.2 \%$ of patients had hypoechoic tumors and $57.1 \%$ of patients had iso/heteroechoic tumors [19]. In another study conducted by Sarkodie et al. on 90 patients, $55.6 \%$ of the patients had fibroids with mixed echo, $24.4 \%$ with isoechoic, and $20 \%$ with hypoechoic pattern [20]. The results of the above studies are different from the results of our study. The ultrasound view of fibromas may be different, but most of them are in the form of symmetric masses with specific margins and they seem hypoechoic and heterogeneous. Calcification or bleeding areas may be hyperechoic, sometimes having cystic degenerescence and seemingly without echo [21].

Most of the patients with prevailing fibroid tumors had high vascularity $(49.3 \%)$ or low vascularity $(46.4 \%)$. In the study conducted by Cho et al. on 24 patients with fibroid tumors, $50 \%$ of the patients were in the low vascularity and $20.8 \%$ were in the high vascularity group [22]. These results are different from the results of our study. However, in the study conducted by Weintraub et al. $42 / 8 \%$ of the patients with fibroids were in the high vascularity and $42 / 8 \%$ were in the mild vascularity group [19] and this is similar to the results of our study.

The average score of prevailing fibroid volume in the first week, first month, third month, sixth month, and the first year of referring compared to the primary volume is $p<0.001$ in all cases. In other words, the decrease of volume of fibroids following uterine artery embolization was statistically significant. The results of the repeated measurement ANOVA test showed that the average scores of fibroid volume over repeated measurements (six time points) were significantly different and descending, and the treatment has proven to be effective.

Changes of uterus volume in multiple tumors were significant over the first week and the third month, and they were not significant in other cases. In other words, effectiveness of treatment has not been proven. This result can be attributed to the small numbers of patients and their decreased number, so that after one year, the number of patients, which was 11, decreased to 3 people (72\% missing). In the study conducted by Firouznia et al. on 101 patients, the volume of the prevailing fibroid was $247 / 3 \pm 315 / 6 \mathrm{~cm}^{3}$ before performing uterine artery embolization. After one year, this volume was reduced to 70/6 $\pm 74 / 2 \mathrm{~cm}^{3}$ and this was statistically significant $(p<0.0001)$ and consistent with the results of our study. Uterus volume in multiple tumors was first $612 / 1 \pm 465 / 3 \mathrm{~cm}^{3}$, and after performing uterine artery embolization, this volume became $354 / 5 \pm 312 / 8 \mathrm{~cm}^{3}$. This was statistically significant ( $p<0.0001$ ), but it is not consistent with the results of our study [13].

In the study conducted by Horhoianu et al. on 30 patients, the average volume of fibroid was reduced from $64 / 27 \pm 77 / 88 \mathrm{~cm}^{3}$ to $46 / 63 \pm 55 / 87 \mathrm{~cm}^{3}$ over 30 days. This was statistically significant $(p<0.001)$ and it is consistent with the results of our study [23].

In the study conducted by Jun et al. on 65 patients, the primary volume of fibroid was $125 \pm 46 \mathrm{~cm}^{3}$ and after six months of performing uterine artery embolization, this volume became $51 \pm 11 \mathrm{~cm}^{3}$ (61\% reduction). This was statistically significant $(p<0.01)$ and consistent with the results of our study [24]. The results of the repeated measurement ANOVA test regarding the relationship between location of prevailing fibroid and decrease of fibroid volume was significant $(p=0.006)$. In other words, in our study, the tumors more prevalent in intramural, submucosal, and transmural areas have had better reactions to treatment.

In the study conducted by Zhou et al., it was observed that the location of fibroids influences the decrease of their volume, so that the tumors in intramural and submucosal areas have better responses to treatment, and this is consistent with the results of our study [25]. Also, in another study conducted by Spies et al. it was observed that the location of fibroids in the uterine wall influences treatment, and this is similar to the result of our study, so that tumors located in the submucosal area have a higher tendency to volume decrease [26].

In the study conducted by Firouznia et al. there was no significant difference between the location of fibroid and decrease of fibroid volume, and this is not consistent with the results of our study. On the other hand, this study showed that the number of fibroid numbers has no effect on decrease of their volume and this is consistent with the results of our study [13].

In another study conducted by Weintraub et al., no significant relationship was found between echogenicity and vascularity of fibroid tumors and the percentage of reduction in their volume, and this is consistent with the results of our study [19].

In the study conducted by Isonishi et al. on 43 patients, 17-month follow-up showed that single and high vascular fibroid tumors have more chance of success in volume reduction by uterine artery embolization treatment [27].

In a study, Kim et al. indicated that low vascularity is a prevailing finding in cervical uterine fibroids, and three months after treatment, these tumors have a disappointing response to treatment by uterine artery embolization [28]. In the study conducted by Tang et al. on 183 patients, it was found that single and low vascular masses have the highest chance of treatment and no long-term recurrence [29]. In another study conducted by Fleischer et al. it was found that high vascular tumors have a higher tendency to volume decrease than low vascular or isovascular tumors [30]. The results of the above studies are not consistent 
with the results of our study. The important limitation of this study was the number of patients and also availability of only one device, which made the study time-consuming.

\section{Conclusions}

It can be certainly stated that uterine artery embolization is an effective treatment for fibroid tumors. In this study, the number of tumors, echogenicity, and vascularity of tumors had no effect on tumor volume reduction, and only location of the masses in the uterine wall had an effect.

\section{Acknowledgments}

The present research article has been extracted from the "Thesis" conducted as a research project funded by the Research Deputy of Ahvaz Jundishapur University of Medical Sciences, Ahvaz, Iran.

The authors declare no conflict of interest.

\section{References}

1. Shirali S, Barari A, Hosseini SA, Khodadi E. Effects of six weeks endurance training and aloe vera supplementation on COX-2 and VEGF levels in mice with breast cancer. Asian Pac J Cancer Prev 2017; 18: 31-36.

2. Rahmani H, Shahriary A, Sheikhi MA, Ebadi A, Davoodzadeh H. Applications of cardiotoxicity in breast cancer: a meta-analysis. Panminerva Med 2017; 59: 90-96.

3. Asadi J, Moradi Binabaj M, Hosseini SA, Khoshnazar AK. The simultaneous effect of valproic acid and gamma radiation on telomerase activity and bax and $\mathrm{BCl}-2$ protein levels in MCF-7 breast cancer cell line. Jundishapur J Nat Pharm Prod 2015; 10: e22818.

4. Parker WH. Etiology, symptomatology, and diagnosis of uterine myomas. Fertil Steril 2007;87: 725-736.

5. Firouznia K, Ghanaati H, Jalali AH, Shakiba M. Uterine artery embolization for treatment of symptomatic fibroids: a review of the evidence. Iran Red Crescent Med J 2013; 15: e16699.

6. Hartmann KE, Birnbaum H, Ben-Hamadi R, et al. Annual costs associated with diagnosis of uterine leiomyomata. Obstet Gynecol 2006; 108: 930-937.

7. Voogt MJ, Arntz MJ, Lohle PN, Willem PTM, Lampmann LE. Uterine fibroid embolisation for symptomatic uterine fibroids: a survey of clinical practice in Europe. Cardiovasc Intervent Radiol 2011; 34: 765-773.

8. Lyon SM, Cavanagh K. Uterine artery embolisation-a treatment alternative for women with fibroids. Aust Fam Physician 2006; 35 : 300-303.

9. Bonduki CE, Feldner Jr PC, Silva Jd, Castro RA, Sartori MG, Girao MJ. Pregnancy after uterine arterial embolization. Clinics (Sao Paulo) 2011; 66: 807-810.

10. Bradley LD. Uterine fibroid embolization: a viable alternative to hysterectomy. Am J Obstet Gynecol 2009; 201: 127-135.

11. Walker WJ, Pelage J. Uterine artery embolisation of symptomatic uterine fibroids: clinical results in 400 women with imaging follow up. BJOG 2002; 109: 1262-1271.

12. Gupta JK, Sinha AS, Lumsden MA, Hickey M. Uterine artery embolization for symptomatic uterine fibroids. Cochrane Database Syst Rev 2006; 25: CD005073.

13. Firouznia K, Ghanaati H, Sanaati M, Jalali AH, Shakiba M. Uterine artery embolization in 101 cases of uterine fibroids: do size, location, and number of fibroids affect therapeutic success and complications? Cardiovasc Intervent Radiol 2008; 31: 521-526.

14. Spies JB, Bruno J, Czeyda-Pommersheim F, Magee ST, Ascher SA, Jha RC. Long-term outcome of uterine artery embolization of leiomyomata. Obstet Gynecol 2005; 106 (5 Pt 1): 933-939.
15. Parthipun A, Taylor J, Manyonda I, Belli A. Does size really matter? Analysis of the effect of large fibroids and uterine volumes on complication rates of uterine artery embolisation. Cardiovasc Intervent Radiol 2010; 33(5): 955-959.

16. Volkers NA, Hehenkamp WJ, Birnie E, de Vries C, Holt C, Ankum WM, Reekers JA. Uterine artery embolization in the treatment of symptomatic uterine fibroid tumors (EMMY trial): periprocedural results and complications. J Vasc Interv Radiol 2006; 17: 471-480.

17. David M, Ebert AD. Treatment of uterine fibroids by embolization - Advantages, disadvantages, and pitfalls. Eur J Obstet Gynecol Reprod Biol 2005; 123: 131-138.

18. Marshburn PB, Matthews ML, Hurst BS. Uterine artery embolization as a treatment option for uterine myomas. Obstet Gynecol Clin North Am 2006; 33: 125-144.

19. Weintraub JL, Romano WJ, Kirsch MJ, Sampaleanu DM, Madrazo BL. Uterine Artery Embolization Sonographic Imaging Findings. J Ultrasound Med 2002; 21: 633-637.

20. Sarkodie BD, Botwe BO, Ofori EK. Uterine fibroid characteristics and sonographic pattern among Ghanaian females undergoing pelvic ultrasound scan: a study at 3-major centres. BMC Womens Health 2016; 16: 10.

21. Dueholm M, Lundorf E, Hansen ES, Ledertoug S, Olesen F. Accuracy of magnetic resonance imaging and transvaginal ultrasonography in the diagnosis, mapping, and measurement of uterine myomas. Am J Obstet Gynecol 2002; 186: 409-415.

22. Cho JY, Kim SH, Kim SY, Moon SK, Li J. Efficacy and Safety of Daily Repeated Sonographically Guided High-Intensity Focused Ultrasound Treatment of Uterine Fibroids Preliminary Study. J Ultrasound Med 2013; 32: 397-406.

23. Horhoianu IA, Horhoianu VV, Joita D, Carstoiu M, Dorobat B. Uterine artery embolization for leioyomas, ultrasonography and angiography aspects. J Med Life 2012; 5: 491.

24. Jun F, Yamin L, Yushun Z, Lijuan W, Xingye W, Hao Q, Wenli G. Effect of selective uterine artery embolization on symptomatic uterine fibroids. J Med Coll PLA 2009; 24: 346-353.

25. Zhou SK, Li SB, Zhang ZS. [Influential factors to therapeutic efficacy of uterine artery embolization in the treatment of uterine fibroids]. Hunan Yi Ke Da Xue Xue Bao 2003; 28: 409-411.

26. Spies JB, Myers ER, Worthington-Kirsch R, Mulgund J, Goodwin S, Mauro M; FIBROID Registry Investigators. The FIBROID Registry: symptom and quality-of-life status 1 year after therapy. Obstet Gynecol 2005; 106: 1309-1318.

27. Isonishi S, Coleman RL, Hirama M, lida Y, Kitai S, Nagase M, Ochi ai K. Analysis of prognostic factors for patients with leiomyoma treated with uterine arterial embolization. Am J Obstet Gynecol 2008; 198: 270-e1.

28. Kim MD, Lee M, Jung DC, Park SI, Lee MS, Won JY, Lee DY, Lee KH. Limited efficacy of uterine artery embolization for cervical leiomyomas. J Vasc Interv Radiol 2012; 23: 236-240.

29. Tang Y, Chen C, Duan H, Ma B, Liu P. Low vascularity predicts favourable outcomes in leiomyoma patients treated with uterine artery embolization. Eur Radiol 2016; 26: 3571-3579.

30. Fleischer AC, Donnelly EF, Campbell MG, Mazer MJ, Grippo D, Lipsitz NL. Three-dimensional color Doppler sonography before and after fibroid embolization. J Ultrasound Med 2000; 19: 701-705.

\section{Address for correspondence}

\section{Azim Motamedfar}

Department of Radiology

Golestan Hospital

Ahvaz Jundishapur University of Medical Sciences

6135733118 Ahvaz, Iran

e-mail: motamedfar-a@ajums.ac.ir

Submitted: 16.09 .2018

Accepted: $\quad 3.03 .2019$ 